\title{
A stabilised mixed finite element method for the Poisson problem based on a three-field formulation
}

\author{
Muhammad Ilyas ${ }^{1} \quad$ Bishnu P. Lamichhane ${ }^{2}$
}

(Received 7 January 2016; revised 8 August 2016)

\begin{abstract}
We present a mixed finite element method for a three-field formulation of the Poisson problem and apply a biorthogonal system leading to an efficient numerical computation. The three-field formulation is similar to the $\mathrm{Hu}$-Washizu formulation for the linear elasticity problem. A parameterised approach is given to stabilise the problem so that its associated bilinear form is coercive on the whole space. Analysis of optimal choices of parameter approximation and numerical examples are provided to evaluate our stabilised form.
\end{abstract}

DOI:10.21914/anziamj.v57i0.10356 gives this article, (c) Austral. Mathematical Soc. 2016. Published September 11, 2016, as part of the Proceedings of the 12th Biennial Engineering Mathematics and Applications Conference. ISSN 1445-8810. (Print two pages per sheet of paper.) Copies of this article must not be made otherwise available on the internet; instead link directly to the DOI for this article. Record comments on this article via http://journal. austms.org. au/ojs/index.php/ANZIAMJ/comment/add/10356/0 


\section{Contents}

1 Introduction

C178

2 A three-field formulation of the Poisson equation

C180

3 Stabilisation methods and well posedness

C182

4 Finite element discretisation

C184

5 Optimal parameter approximation

C186

6 Numerical Examples

C187

6.1 Analytic solution

C187

6.2 Re-entrant corner

7 Conclusion

C190

References

C191

\section{Introduction}

We introduce a mixed finite element method for solving the Poisson equation. We use the gradient of the solution of the Poisson equation as a new unknown and write an additional variational equation in terms of a Lagrange multiplier. This gives two additional vector unknowns: the gradient of the solution and the Lagrange multiplier. In a discrete setting, we choose bases for the gradient space and Lagrange multiplier space so that they form a biorthogonal system.

There are many mixed finite element methods for the Poisson equation $[1,2,3$, 7]. However, all of them are based on the two-field formulation of the Poisson equation and not suitable for the application of a biorthogonal system. Since the application of a biorthogonal system needs different spaces for the solution, 
the gradient of the solution and the Lagrange multiplier, we need a three-field formulation. Moreover, a biorthogonal system with three-field formulation leads to a symmetric formulation for efficient numerical computation [6]. In Section 2 we introduce our Poisson problem and its mixed formulation. A similar three-field formulation, known as the $\mathrm{Hu}$-Washizu formulation, is widely used in linear elasticity problems, with displacement, stress and strain as three unknowns [2] (e.g., Reissner-Mindlin plates [4], linear elasticity [5] and incompressible elasticity [6]).

The difficulty of the three-field formulation is that the associated bilinear form of the Poisson problem is not coercive on the whole space. We need to consider this difficulty in developing a finite element method for this problem. In Section 3 we propose an approach to stabilise the bilinear form so that it is coercive on the whole space. The stabilised form is parameterised to find an optimal parameter to improve the accuracy of the solution.

In Section 4 we give a discrete formulation for the stabilised form with corresponding spaces and biorthogonal bases. Furthermore, in Section 5 we give the calculation of optimal parameter approximation for our stabilised form. A numerical approximation of the solution is obtained by condensing out the degrees of freedom associated with the gradient and the Lagrange multiplier, so that we arrive at a reduced system of equations which is easy to solve. The gradient of the solution and the Lagrange multiplier is computed as a post-processing step. In Section 6 we evaluate the error of the solution in $\mathrm{L}^{2}$ and $\mathrm{H}^{1}$-norms and the error of the gradient in the $\mathrm{L}^{2}$-norm to assess our optimal parameter choice. 


\section{A three-field formulation of the Poisson equation}

Let $\mathrm{V}=\mathrm{H}_{0}^{1}(\Omega)$ and $\mathrm{L}=\left[\mathrm{L}^{2}(\Omega)\right]^{\mathrm{d}}$ for $\mathrm{d} \in\{2,3\}$. Given $\mathrm{f} \in \mathrm{L}^{2}(\Omega)$, we start with the minimisation problem for the Poisson problem:

$$
\underset{v \in \mathrm{V}}{\operatorname{argmin}} \frac{1}{2}\|\nabla v\|_{0, \Omega}^{2}-\ell(v)
$$

where $\ell(v)=\int_{\Omega} f v d x$.

Our mixed formulation is obtained by introducing $\sigma=\nabla \mathfrak{u}$ so that the minimisation problem (1) is rewritten as

$$
\underset{\substack{(\mathfrak{u}, \boldsymbol{\sigma}) \in V \times \mathrm{V} \times \mathrm{L} \\ \sigma=\nabla \mathfrak{u}}}{\operatorname{argmin}} \frac{1}{2}\|\sigma\|_{0, \Omega}^{2}-\ell(v) \text {. }
$$

The norm for the product space $\mathrm{V} \times \mathrm{L}$ is defined by

$$
\|(u, \sigma)\|_{V \times L}=\sqrt{\|\sigma\|_{0, \Omega}^{2}+\|u\|_{1, \Omega}^{2}}
$$

for $(u, \sigma) \in \mathrm{V} \times \mathrm{L}$. We write a weak variational equation for $\sigma=\nabla \mathrm{u}$ using the Lagrange multiplier space $M=L$ to obtain the saddle-point problem of the minimisation problem (2). The saddle-point formulation is to find $(u, \sigma, \varphi) \in \mathrm{V} \times \mathrm{L} \times \mathrm{M}$ such that

$$
\begin{aligned}
\widetilde{a}[(u, \sigma),(v, \tau)]+b[(v, \tau), \varphi] & =\ell(v), \quad(v, \tau) \in V \times L, \\
b[(u, \sigma), \psi] & =0, \quad \psi \in M,
\end{aligned}
$$

where

$$
\begin{aligned}
\widetilde{\mathrm{a}}[(\mathrm{u}, \sigma),(v, \tau)] & =\int_{\Omega} \sigma \cdot \tau \mathrm{d} x, \\
\mathrm{~b}[(\mathrm{u}, \sigma), \psi] & =\int_{\Omega}(\sigma-\nabla \mathfrak{u}) \cdot \psi \mathrm{d} x, \\
\ell(v) & =\int_{\Omega} f v \mathrm{~d} x .
\end{aligned}
$$


To show that saddle-point problem (3) has a unique solution, we need to show that the following three conditions of well-posedness are satisfied.

1. The linear form $\ell(\cdot)$, the bilinear forms $\widetilde{a}[\cdot, \cdot]$ and $b[\cdot, \cdot]$ are continuous on the spaces in which they are defined.

2. The bilinear form $\widetilde{a}[\cdot, \cdot]$ is coercive on the kernel space $\mathbf{K}$ defined as

$$
K=\{(u, \sigma) \in V \times L: b[(u, \sigma), \psi]=0, \text { for all } \psi \in M\} .
$$

3. The bilinear form $\mathrm{b}[\cdot, \cdot]$ satisfies the inf-sup condition

$$
\inf _{\psi \in M} \sup _{(v, \tau) \in V \times L} \frac{b[(\nu, \tau), \psi]}{\|(v, \tau)\|_{V \times L}\|\psi\|_{M}} \geqslant \gamma, \quad \gamma>0 .
$$

In the saddle-point formulation (3), the bilinear form $\widetilde{\mathbf{a}}[\cdot, \cdot]$ is not coercive on the whole space $\mathrm{V} \times \mathrm{L}$. It is only coercive on the kernel subspace $\mathrm{K} \subset \mathrm{V} \times \mathrm{L}$. The coercivity of the bilinear form $\widetilde{a}[\cdot, \cdot]$ on the subspace $K$ follows from the Poincaré inequality,

$$
|\widetilde{a}[(u, \sigma),(u, \sigma)]|=\|\sigma\|_{0, \Omega}^{2} \geqslant C\|(u, \sigma)\|_{V \times L}^{2} \quad \text { as } \sigma=\nabla u \text { on } K .
$$

Unfortunately, in the discrete formulation, it is not easy to select an appropriate kernel space to satisfy the coercivity condition. We need to consider this difficulty in developing a finite element method for this problem. It is easier to develop a finite element method if the bilinear form $\widetilde{a}(\cdot, \cdot)$ is coercive on the whole space $\mathrm{V} \times \mathrm{L}$. One way to make the bilinear form $\widetilde{\mathrm{a}}[\cdot, \cdot]$ coercive on the whole space $V \times L$ is to stabilise it.

In this article, we propose an approach to stabilise the bilinear form $\widetilde{a}[\cdot, \cdot]$ so that it is coercive on the whole space $V \times$ L. To get a consistent stabilisation, our approach modifies the bilinear form $\widetilde{a}[\cdot, \cdot]$ by adding a stabilisation term. 


\section{Stabilisation methods and well posedness}

In this section, we define the stabilisation method for the bilinear form $\widetilde{a}[\cdot, \cdot]$ so that it is coercive on the whole space $\mathrm{V} \times \mathrm{L}$ and prove that conditions of well-posedness are satisfied [2,3]. Furthermore, we introduce an additional parameter for our approach. We give calculations for continuity and coercivity constant as functions of this parameter. These calculations are used to get optimal parameter approximation according to the theory of saddle point problems, defined in Section 5.

We modify the bilinear form $\widetilde{a}[\cdot, \cdot]$ so that it not only contains $\sigma$, but also contains $\nabla \mathfrak{u}$, so we can control both terms in the coercivity condition. The bilinear form is modified as

$$
\mathrm{a}[(\mathrm{u}, \sigma),(v, \tau)]=\mathrm{r} \int_{\Omega} \sigma \cdot \tau \mathrm{d} x+(1-\mathrm{r}) \int_{\Omega} \nabla \mathrm{u} \cdot \nabla v \mathrm{~d} x, \quad 0 \leqslant \mathrm{r} \leqslant 1 .
$$

Thus our problem is to find $(u, \sigma, \varphi) \in \mathrm{V} \times \mathrm{L} \times \mathrm{M}$ such that

$$
\begin{aligned}
\mathrm{a}[(\mathrm{u}, \sigma),(\nu, \tau)]+\mathrm{b}[(\nu, \tau), \varphi] & =\ell(v), \quad(\nu, \tau) \in \mathrm{V} \times \mathrm{L}, \\
\mathrm{b}[(\mathrm{u}, \sigma), \psi] & =0, \quad \psi \in \mathrm{M},
\end{aligned}
$$

When $r=1$, we have our original bilinear form and when $r=0$ the system is uncoupled. Thus we set the parameter $r$ as $0<r<1$. Moreover, $\mathrm{a}[(u, \sigma),(v, \tau)]=\widetilde{a}[(u, \sigma),(v, \tau)]$ on kernel space $\mathrm{K}$.

In the saddle-point formulation (5), we need to show that the conditions of well-posedness are satisfied. The bilinear form $\mathrm{b}[\cdot, \cdot]$ and the linear form $\ell(\cdot)$ are continuous by the Cauchy-Schwarz inequality. The continuity of the bilinear form $a[\cdot, \cdot]$ is proved in the following lemma

Lemma 1. The bilinear form a $[\cdot, \cdot]$ is continuous on $\mathrm{V} \times \mathrm{L}$; that is, there exists $\mathrm{C}>0$ such that

$$
|\mathrm{a}[(\mathrm{u}, \sigma),(v, \tau)]| \leqslant \mathrm{C}\|(\mathrm{u}, \sigma)\|_{V \times \mathrm{L}}\|(v, \tau)\|_{V \times \mathrm{L}}, \quad(\mathrm{u}, \sigma),(v, \tau) \in \mathrm{V} \times \mathrm{L},
$$

where $C=\sqrt{2} \max (r, 1-r)$. 


\section{Proof:}

$$
\begin{aligned}
|\mathrm{a}[(\mathrm{u}, \sigma),(v, \tau)]| & \leqslant \mathrm{r}\|\sigma\|_{0, \Omega}\|\tau\|_{0, \Omega}+(1-\mathrm{r})\|\nabla \mathrm{u}\|_{0, \Omega}\|\nabla v\|_{0, \Omega} \\
& =\mathrm{C}\|\mathrm{u}, \sigma\|_{V \times \mathrm{L}}\|v, \tau\|_{V \times \mathrm{L}},
\end{aligned}
$$

where $C=\sqrt{2} \max (r, 1-r)$ depends only on $r$.

It remains to show that bilinear form $a[\cdot, \cdot]$ is coercive on the whole space and bilinear form $\mathrm{b}[\cdot, \cdot]$ satisfies the inf-sup condition (4).

Lemma 2. The bilinear form a $[\cdot, \cdot]$ is coercive on $\mathrm{V} \times \mathrm{L}$; that is, there exists $\alpha>0$ such that

$$
|a[(u, \sigma),(u, \sigma)]| \geqslant \alpha\|(u, \sigma)\|_{V \times L}^{2}, \quad(u, \sigma) \in V \times L
$$

where $\frac{1}{\alpha}=\max \left(\frac{1}{r}, \frac{\mathrm{c}^{2}+1}{1-r}\right)$ and $\mathrm{c}$ is the Poincaré constant with $\|\mathrm{u}\|_{0, \Omega} \leqslant$ c $\|\nabla \mathrm{u}\|_{0, \Omega}$.

Proof: We have $\|u\|_{1, \Omega}^{2}=\|u\|_{0, \Omega}^{2}+\|\nabla u\|_{0, \Omega}^{2} \leqslant\left(c^{2}+1\right)\|\nabla u\|_{0, \Omega}^{2}$. Therefore,

$$
\begin{aligned}
\|(u, \sigma)\|_{V \times \mathrm{L}}^{2} & =\|\sigma\|_{0, \Omega}^{2}+\|\mathrm{u}\|_{1, \Omega}^{2} \\
& \leqslant\|\sigma\|_{0, \Omega}^{2}+\left(c^{2}+1\right)\|\nabla u\|_{0, \Omega}^{2} \\
& =\frac{1}{r}\left(r\|\sigma\|_{0, \Omega}^{2}\right)+\frac{c^{2}+1}{1-r}\left((1-r)\|\nabla u\|_{0, \Omega}^{2}\right) \\
& \leqslant \frac{1}{\alpha}\left|a_{1}[(u, \sigma),(u, \sigma)]\right|,
\end{aligned}
$$

where $\mathrm{c}$ is the Poincaré inequality constant and $\frac{1}{\alpha}=\max \left(\frac{1}{r}, \frac{c^{2}+1}{1-r}\right)$ depends on $r$ and $c$. 
Lemma 3. The bilinear form $\mathrm{b}[\cdot, \cdot]$ satisfies the inf-sup condition; that is, there exists $\gamma>0$ such that

$$
\inf _{\psi \in M} \sup _{(v, \tau) \in V \times L} \frac{b[(\nu, \tau), \psi]}{\|(\nu, \tau)\|_{V \times L}\|\psi\|_{M}} \geqslant \gamma
$$

Proof: By choosing $v=0$,

$$
\sup _{(\nu, \tau) \in V \times L} \frac{b[(v, \tau), \psi]}{\|(\nu, \tau)\|_{V \times L}} \geqslant \sup _{\tau \in L} \frac{\int_{\Omega} \tau \cdot \psi d x}{\|\tau\|_{0}}=\|\psi\|_{0},
$$

and taking infimum from both sides for $\psi \in M$, we get the desired inequality.

Therefore, by the theory of the saddle point problem [2,3], there exists a unique solution of (5), that is, $(\mathfrak{u}, \sigma, \varphi) \in \mathrm{V} \times \mathrm{L} \times \mathrm{M}$ and the solution is stable with respect to the right hand side such that

$$
\|u\|_{1, \Omega}+\|\sigma\|_{0, \Omega}+\|\varphi\|_{0, \Omega} \leqslant C\|\ell\|_{0, \Omega} .
$$

\section{Finite element discretisation}

Let $\mathcal{T}_{h}$ be a quasi-uniform triangulation of the polygonal domain $\Omega$. We use the standard linear finite element space $V_{h} \subset H^{1}(\Omega)$ defined on the triangulation $\mathcal{T}_{h}$, where

$$
V_{h}:=\left\{v \in C^{0}(\Omega):\left.v\right|_{T} \in \mathcal{P}_{1}(T), T \in \mathcal{T}_{h}\right\} .
$$

Let $V_{h}^{0}=V_{h} \cap H_{0}^{1}(\Omega)$. The finite element space for the gradient of the solution is $L_{h}=\left[V_{h}\right]^{2}$. Let $\left\{\rho_{1}, \rho_{2}, \ldots, \rho_{N}\right\}$ be the finite element basis for $V_{h}$. Starting with the standard basis for $V_{h}$, we construct a space $Q_{h}$ spanned by 
the basis $\left\{\mu_{1}, \mu_{2}, \ldots, \mu_{N}\right\}$ so that the basis functions of $V_{h}$ and $Q_{h}$ satisfy the biorthogonality condition

$$
\int_{\Omega} \rho_{i} \mu_{j} d x=c_{j} \delta_{i j}, \quad c_{j} \neq 0, \quad 1 \leqslant i, j \leqslant N,
$$

where $\delta_{i j}$ is the Kronecker symbol, and $c_{j}$ a scaling factor. Therefore, the sets of basis functions of $V_{h}$ and $Q_{h}$ form a biorthogonal system. The basis functions of $Q_{h}$ are constructed locally on a reference element $\uparrow \in \mathcal{T}_{h}$ so that the basis functions of $V_{h}$ and $Q_{h}$ have the same support, and in each element the sum of all the basis functions of $Q_{h}$ is one $[6,4]$. We let $M_{h}=\left[Q_{h}\right]^{2}$, thus our problem is to find $\left(u_{h}, \sigma_{h}, \varphi_{h}\right) \in V_{h}^{0} \times L_{h} \times M_{h}$ such that

$$
\begin{aligned}
\mathrm{a}\left[\left(\mathrm{u}_{\mathrm{h}}, \sigma_{\mathrm{h}}\right),\left(v_{\mathrm{h}}, \tau_{\mathrm{h}}\right)\right]+\mathrm{b}\left[\left(v_{\mathrm{h}}, \tau_{\mathrm{h}}\right), \varphi_{\mathrm{h}}\right] & =\ell\left(v_{\mathrm{h}}\right), \quad\left(v_{\mathrm{h}}, \tau_{\mathrm{h}}\right) \in \mathrm{V}_{\mathrm{h}}^{0} \times \mathrm{L}_{\mathrm{h}}, \\
\mathrm{b}\left[\left(\mathrm{u}_{\mathrm{h}}, \sigma_{\mathrm{h}}\right), \psi_{\mathrm{h}}\right] & =0, \quad \psi_{\mathrm{h}} \in \mathrm{M}_{\mathrm{h}},
\end{aligned}
$$

To present an algebraic formulation of the problem, we use the same notation for the vector representation of the solution and the solution $\left(\mathfrak{u}_{h}, \sigma_{h}, \varphi_{h}\right)$ as elements in $V_{h}^{0} \times L_{h} \times M_{h}$. Let $M, D, A, B$ and $C$ be the matrices associated with bilinear forms $\int_{\Omega} \sigma_{h} \cdot \tau_{h} d x, \int_{\Omega} \tau_{h} \cdot \varphi_{h} d x, \int_{\Omega} \nabla u_{h} \cdot \nabla v_{h} d x, \int_{\Omega} \nabla u_{h}$. $\varphi_{\mathrm{h}} \mathrm{d} x$ and $\int_{\Omega} \sigma_{\mathrm{h}} \cdot \nabla v_{\mathrm{h}} \mathrm{dx}$, respectively. Let $\ell$ be an $\mathrm{n}$-component vector with $\left\{\ell_{i}=\int_{\Omega} f \rho_{i} d x\right\}_{i=1}^{N}$. Then the algebraic formulation of the problem is

$$
\left[\begin{array}{ccc}
(1-r) A & 0 & -B \\
0 & r M & D \\
-B^{T} & D & 0
\end{array}\right]\left[\begin{array}{l}
u_{h} \\
\sigma_{h} \\
\varphi_{h}
\end{array}\right]=\left[\begin{array}{l}
\ell \\
0 \\
0
\end{array}\right]
$$

where the first two equations of (7) correspond to first equation of (6), by setting $\sigma_{h}=0$ and $v_{h}=0$, respectively. After statically condensing out degrees of freedom associated with $\sigma_{h}$ and $\varphi_{h}$ in the above matrix equation, we have the system

$$
\left[(1-r) A+r\left(B D^{-1} M D^{-1} B^{\top}\right)\right] u_{h}=\ell .
$$


Due to the choice of a biorthogonal system, matrix D is diagonal. As a result, the statically condensed system matrix is sparse. Finally, we approximate the gradient by

$$
\sigma_{h}=D^{-1} B^{\top} u_{h}
$$

\section{Optimal parameter approximation}

In this section we give optimal parameter approximations for both our stabilised forms based on the bound of the error between $(u, \sigma)$ and $\left(u_{h}, \sigma_{h}\right)$ in terms of best approximation errors

$$
\inf _{\left(v_{h}, \tau_{h}\right) \in V_{h} \times L_{h}}\left\|(u, \sigma)-\left(v_{h}, \tau_{h}\right)\right\|_{V \times L} \quad \text { and } \quad \inf _{\psi_{h} \in M_{h}}\left\|\varphi-\psi_{h}\right\|_{M},
$$

which are an extension of Céa's Lemma from the classical finite element method to the mixed finite element method [2].

Lemma 4. Suppose that well-posedness conditions are satisfied. In addition, suppose $(\mathrm{u}, \sigma)$ and $\left(\mathfrak{u}_{\mathrm{h}}, \sigma_{\mathrm{h}}\right)$ are the solutions of the variational problem in $\mathrm{V} \times \mathrm{L}$ and $\mathrm{V}_{\mathrm{h}} \times \mathrm{L}_{\mathrm{h}}$, respectively. Then $\left\|(\mathrm{u}, \sigma)-\left(\mathfrak{u}_{\mathrm{h}}, \sigma_{\mathrm{h}}\right)\right\|_{\mathrm{V} \times \mathrm{L}}$ is bounded from above by

$$
\left(1+\frac{C_{a}}{\alpha}\right) \inf _{\left(v_{h}, \tau_{h}\right) \in V_{h} \times L_{h}}\left\|(u, \sigma)-\left(v_{h}, \tau_{h}\right)\right\|_{V \times L}+\frac{C_{b}}{\alpha} \inf _{\psi_{h} \in M_{h}}\left\|\varphi-\psi_{h}\right\|_{M},
$$

where $\mathrm{C}_{\mathrm{a}}$ and $\mathrm{C}_{\mathrm{b}}$ are the continuity constants for bilinear forms $\mathrm{a}[\cdot, \cdot]$ and $\mathrm{b}[\cdot, \cdot]$, respectively, and $\alpha$ is the coercivity constant of $\mathrm{a}[\cdot, \cdot]$ [2].

The main point of Lemma 4 is that the error $(u, \sigma)-\left(u_{h}, \sigma_{h}\right)$ is bounded in terms of the best approximation errors by assuming boundedness of the bilinear forms $a[\cdot, \cdot]$ and $b[\cdot, \cdot]$ and the coercivity of $a[\cdot, \cdot]$. In order to find the optimal continuity and coercivity constant in Lemma 4, we review approximation in Section 4 for our approach. 
To get a better error estimate, we minimise $\frac{C_{a}}{\alpha}$ with respect to $r$ in Lemma 4 . From Lemmas 1 and 2,

$$
\begin{aligned}
\underset{r}{\operatorname{argmin}} \frac{C_{a}}{\alpha} & =\underset{r}{\operatorname{argmin}}\left\{\sqrt{2} \max (r, 1-r) \cdot \max \left(\frac{1}{r}, \frac{c^{2}+1}{1-r}\right)\right\} \\
& = \begin{cases}\frac{\sqrt{2}(1-r)}{r}, & \text { if } 0<r<\frac{1}{c^{2}+2}, \\
\left(c^{2}+1\right) \sqrt{2}, & \text { if } \frac{1}{c^{2}+2} \leqslant r \leqslant \frac{1}{2}, \\
\frac{\left(c^{2}+1\right) \sqrt{2} r}{1-r}, & \text { if } \frac{1}{2}<r<1,\end{cases}
\end{aligned}
$$

where the Poincaré constant $\mathrm{c}$ depends on the domain of the problem.

\section{$6 \quad$ Numerical Examples}

In this section we assess our optimal parameter choice by evaluating the solution errors $\left\|\mathfrak{u}-\mathfrak{u}_{\mathfrak{h}}\right\|_{0, \Omega}$ and $\left\|\mathfrak{u}-\mathfrak{u}_{\mathfrak{h}}\right\|_{1, \Omega}$, the gradient of the solution error $\left\|\sigma-\sigma_{h}\right\|_{0, \Omega}$ and the Lagrange multiplier error $\left\|\varphi-\varphi_{h}\right\|_{0, \Omega}$. We provide numerical results for our approach with several values of $r$. Mitchell [8] discusses all our test problems.

\subsection{Analytic solution}

For this problem, the function $f$ is derived from the exact solution $u=$ $2^{4 a} x^{a}(1-x)^{a} y^{a}(1-y)^{a}$, where parameter a determines the degree of polynomial solution and it should be chosen to be large enough so that the highest order finite elements to be used will not give the exact solution. This problem has Dirichlet boundary conditions on $\partial \Omega$ and is defined on $\Omega=[0,1]^{2}$. This is a well behaved problem with a smooth solution that has no trouble spots. In this example, we set parameter $a=5$ and mesh initialisation is given in the left image of Figure 1. 
Figure 1: Mesh initialisation.

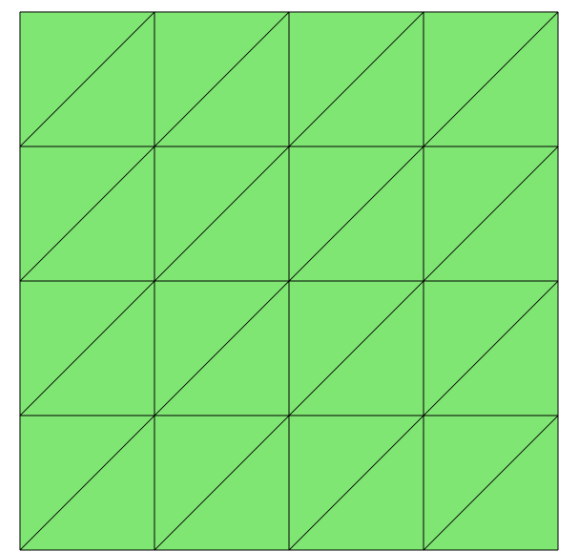

(a) Analytic solution mesh.

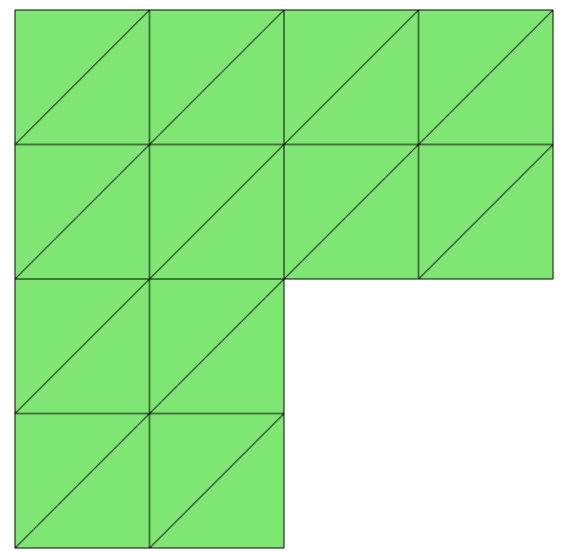

(b) Re-entrant corner mesh.

Table 1: Discretisation error $\left\|\mathfrak{u}-\mathfrak{u}_{\mathfrak{h}}\right\|_{0, \Omega}$.

\begin{tabular}{r|cc|cc|cc|cc}
\multirow{2}{*}{ no. elements } & \multicolumn{2}{|c|}{$r=0.01$} & \multicolumn{2}{c|}{$r=1 / 3$} & \multicolumn{2}{c|}{$r=1 / 2$} & \multicolumn{2}{c}{$r=0.90$} \\
& error & rate & error & rate & error & rate & error & rate \\
\hline 32 & 0.123 & 2.365 & 0.096 & 3.270 & 0.082 & 3.835 & 0.341 & 2.934 \\
128 & 0.045 & 1.464 & 0.023 & 2.043 & 0.013 & 2.646 & 0.071 & 2.272 \\
512 & 0.013 & 1.764 & 0.005 & 2.348 & 0.002 & 2.566 & 0.016 & 2.160 \\
2048 & 0.003 & 1.935 & 0.001 & 2.201 & 0.001 & 1.927 & 0.004 & 2.064 \\
8192 & 0.001 & 1.984 & 0.000 & 2.061 & 0.000 & 1.955 & 0.001 & 2.016
\end{tabular}

Since the Poincare inequality constant for this domain is one, our choice of $r$ depends on (8) and so $\frac{1}{3} \leqslant r \leqslant \frac{1}{2}$ are optimal values of $r$ from Lemma 4 . Tables 1, 2 and 3 give the errors and the rates of convergence for $\left\|\mathfrak{u}-\mathfrak{u}_{h}\right\|_{0, \Omega}$, $\left\|u-u_{h}\right\|_{1, \Omega}$, and $\left\|\sigma-\sigma_{h}\right\|_{0, \Omega}$, respectively, for $r=0.01,1 / 3,1 / 2,0.90$.

Tables 1, 2 and 3 show that our optimal parameter choices give better approximations of the $\mathrm{L}^{2}$ error. For both $\left\|\mathrm{u}-\mathfrak{u}_{\mathfrak{h}}\right\|_{0, \Omega}$ and $\left\|\sigma-\sigma_{h}\right\|_{0, \Omega}$ error rates converge to two and for $\left\|\mathfrak{u}-\mathfrak{u}_{\mathfrak{h}}\right\|_{1, \Omega}$ the error rate converges to one. 
Table 2: Discretisation error $\left\|\mathfrak{u}-\mathfrak{u}_{\mathfrak{h}}\right\|_{1, \Omega}$.

\begin{tabular}{r|cc|cc|cc|cc}
\multirow{2}{*}{ no. elements } & \multicolumn{2}{|c|}{$r=0.01$} & \multicolumn{2}{c|}{$r=1 / 3$} & \multicolumn{2}{c|}{$r=1 / 2$} & \multicolumn{2}{c}{$r=0.90$} \\
& error & rate & error & rate & error & rate & error & rate \\
\hline 32 & 1.083 & 1.928 & 1.087 & 2.398 & 1.251 & 2.506 & 5.531 & 1.461 \\
128 & 0.695 & 0.641 & 0.674 & 0.690 & 0.721 & 0.795 & 1.464 & 1.917 \\
512 & 0.353 & 0.978 & 0.350 & 0.946 & 0.362 & 0.994 & 0.448 & 1.709 \\
2048 & 0.176 & 1.001 & 0.176 & 0.992 & 0.178 & 1.026 & 0.188 & 1.255 \\
8192 & 0.088 & 1.001 & 0.088 & 0.999 & 0.088 & 1.009 & 0.090 & 1.068
\end{tabular}

Table 3: Discretisation error $\left\|\sigma-\sigma_{h}\right\|_{0, \Omega}$.

\begin{tabular}{r|cc|cc|cc|cc}
\multirow{2}{*}{ no. elements } & \multicolumn{2}{|c|}{$r=0.01$} & \multicolumn{2}{c|}{$r=1 / 3$} & \multicolumn{2}{c|}{$r=1 / 2$} & \multicolumn{2}{c}{$r=0.90$} \\
& error & rate & error & rate & error & rate & error & rate \\
\hline 32 & 1.408 & 0.902 & 1.323 & 1.286 & 1.255 & 1.590 & 1.020 & 2.857 \\
128 & 0.752 & 0.905 & 0.601 & 1.139 & 0.013 & 1.340 & 0.156 & 2.707 \\
512 & 0.257 & 1.549 & 0.173 & 1.796 & 0.123 & 2.007 & 0.033 & 2.246 \\
2048 & 0.071 & 1.861 & 0.044 & 1.981 & 0.029 & 2.070 & 0.009 & 1.952 \\
8192 & 0.018 & 1.963 & 0.011 & 1.999 & 0.007 & 2.023 & 0.004 & 1.990
\end{tabular}

\subsection{Re-entrant corner}

For this problem, the function $\mathrm{f}$ is derived from the exact solution $\mathrm{u}=$ $r^{\alpha} \sin (\alpha \theta)$, where $r=\sqrt{x^{2}+y^{2}}$ and $\theta=\tan ^{-1}\left(\frac{y}{x}\right)$. This problem has Dirichlet boundary conditions and is defined on $\Omega=(-1,1)^{2}$ with a section removed from the clockwise side of the positive $x$-axis. The point $(0,0)$ is a source of the singularities in the solution. In particular, for a corner with an angle $\omega$, the solution behaves like $r^{\alpha}$, where $r$ is the distance from the corner and $\alpha=\frac{\pi}{\omega}$. In this example, we set the parameter $\alpha=\frac{2}{3}$ so that $\Omega=(-1,1)^{2} \backslash\{[0,1) \times(-1,0]\}$ and the mesh initialisation is given in the right image of Figure 1.

The Poincaré inequality constant is two. Thus our choice of $r$ depends on (8) 
Table 4: Discretisation error $\left\|\mathfrak{u}-\mathfrak{u}_{\mathrm{h}}\right\|_{0, \Omega}$.

\begin{tabular}{r|cc|cc|cc|cc}
\multirow{2}{*}{ no. elements } & \multicolumn{2}{|c|}{$r=0.01$} & \multicolumn{2}{c|}{$r=1 / 6$} & \multicolumn{2}{c|}{$r=1 / 2$} & \multicolumn{2}{c}{$r=0.90$} \\
& error & rate & error & rate & error & rate & error & rate \\
\hline 24 & 0.016 & 1.244 & 0.012 & 1.413 & 0.010 & 1.447 & 0.029 & 1.015 \\
96 & 0.006 & 1.269 & 0.005 & 1.408 & 0.004 & 1.506 & 0.012 & 1.275 \\
384 & 0.003 & 1.286 & 0.002 & 1.388 & 0.002 & 1.488 & 0.005 & 1.301 \\
1536 & 0.001 & 1.298 & 0.001 & 1.368 & 0.001 & 1.449 & 0.002 & 1.309 \\
6144 & 0.000 & 1.308 & 0.000 & 1.354 & 0.000 & 1.413 & 0.001 & 1.315
\end{tabular}

Table 5: Discretisation error $\left\|\mathfrak{u}-\mathfrak{u}_{\mathrm{h}}\right\|_{1, \Omega}$.

\begin{tabular}{r|cc|cc|cc|cc}
\multirow{2}{*}{ no. elements } & \multicolumn{2}{|c|}{$r=0.01$} & \multicolumn{2}{c|}{$r=1 / 6$} & \multicolumn{2}{c|}{$r=1 / 2$} & \multicolumn{2}{c}{$r=0.90$} \\
& error & rate & error & rate & error & rate & error & rate \\
\hline 24 & 0.177 & 0.630 & 0.178 & 0.627 & 0.191 & 0.613 & 0.299 & 0.507 \\
96 & 0.114 & 0.636 & 0.115 & 0.634 & 0.124 & 0.630 & 0.192 & 0.638 \\
384 & 0.073 & 0.645 & 0.074 & 0.645 & 0.079 & 0.644 & 0.122 & 0.651 \\
1536 & 0.046 & 0.653 & 0.047 & 0.652 & 0.050 & 0.653 & 0.078 & 0.658 \\
6144 & 0.029 & 0.658 & 0.030 & 0.658 & 0.032 & 0.658 & 0.049 & 0.662
\end{tabular}

and so $\frac{1}{6} \leqslant r \leqslant \frac{1}{2}$ are the optimal values of $r$ from Lemma 4. Tables 4,5 and 6 give the errors and the rates of convergence for $\left\|\mathfrak{u}-\mathfrak{u}_{h}\right\|_{0, \Omega},\left\|u-u_{h}\right\|_{1, \Omega}$ and $\left\|\sigma-\sigma_{h}\right\|_{0, \Omega}$, respectively, for $r=0.01,1 / 6,1 / 2,0.90$.

Tables 4, 5 and 6 show that our optimal parameter choices give better approximations on $L^{2}$ error. The error rate for $\left\|u-u_{h}\right\|_{0, \Omega}$ converges to $4 / 3$ and for both $\left\|u-u_{h}\right\|_{1, \Omega}$ and $\left\|\sigma-\sigma_{h}\right\|_{0, \Omega}$ error rates converge to $2 / 3$.

\section{Conclusion}

We describe a parameterised approach to stabilise the bilinear form of a threefield formulation Poisson problem, so that it is coercive on the whole space. 
Table 6: Discretisation error $\left\|\sigma-\sigma_{h}\right\|_{0, \Omega}$.

\begin{tabular}{r|cc|cc|cc|cc}
\multirow{2}{*}{ no. elements } & \multicolumn{2}{|c|}{$r=0.01$} & \multicolumn{2}{|c|}{$r=1 / 6$} & \multicolumn{2}{c|}{$r=1 / 2$} & \multicolumn{2}{c}{$r=0.90$} \\
& error & rate & error & rate & error & rate & error & rate \\
\hline 24 & 0.156 & 0.665 & 0.151 & 0.681 & 0.143 & 0.699 & 0.153 & 0.679 \\
96 & 0.098 & 0.660 & 0.095 & 0.665 & 0.090 & 0.672 & 0.097 & 0.665 \\
384 & 0.062 & 0.663 & 0.060 & 0.665 & 0.057 & 0.668 & 0.061 & 0.665 \\
1536 & 0.039 & 0.665 & 0.038 & 0.666 & 0.036 & 0.667 & 0.038 & 0.666 \\
6144 & 0.025 & 0.666 & 0.024 & 0.666 & 0.022 & 0.667 & 0.024 & 0.666
\end{tabular}

We calculate the optimal parameter based on an extension of Céa's Lemma for mixed finite element problems, approximated from the continuity and coercivity condition of the associated bilinear form. Numerical examples show that our choice of optimal parameter gives better $\mathrm{L}^{2}$-error for the solution than other choices of parameter.

Acknowledgements We are grateful to all referees for their comments and suggestions to improve the quality of the article.

\section{References}

[1] P. B. Bochev and C. R. Dohrmann. A computational study of stabilized, low-order $\mathrm{C}^{0}$ finite element approximations of Darcy equations. Comput. Mech. 38(4):323-333, 2006. doi:10.1007/s00466-006-0036-y C178

[2] D. Braess. Finite Elements: Theory, Fast Solvers, and Applications in Solid Mechanics. Cambridge University Press, 3rd edition, 2007. doi:10.1017/CBO9780511618635 C178, C179, C182, C184, C186

[3] S. C. Brenner and L. R. Scott. The Mathematical Theory of Finite Element Methods. Springer, New York, 3rd edition edition, 2008. doi:10.1007/978-0-387-75934-0 C178, C182, C184 
[4] B. P. Lamichhane. Two simple finite element methods for Reissner-Mindlin plates with clamped boundary condition. Appl. Numer. Math. 72:91-98, 2013. doi:10.1016/j.apnum.2013.04.005 C179, C185

[5] B. P. Lamichhane, A. T. McBride, and B. D. Reddy. A finite element method for a three-field formulation of linear elasticity based on biorthogonal systems. Comput. Method. Appl. Mech. Eng. 258:109-117, 2013. doi:10.1016/j.cma.2013.02.008 C179

[6] B. P. Lamichhane and E. P. Stephan. A symmetric mixed finite element method for nearly incompressible elasticity based on biorthogonal systems. Numer. Meth. Part. D. E. 28(4):1336-1353, 2012. doi:10.1002/num.20683 C179, C185

[7] S. Micheletti and R. Sacco. Dual-primal mixed finite elements for elliptic problems. Comput. Method. Appl. Mech. Eng. 17(2):137-151, 2001. doi:10.1002/1098-2426(200103)17:2<137::AID-NUM4>3.0.CO;2-0 C178

[8] W. F. Mitchell. A collection of 2D elliptic problems for testing adaptive grid refinement algorithms. Appl. Math. Comput. 220:350-364, 2013. doi:10.1016/j.amc.2013.05.068 C187

\section{Author addresses}

1. Muhammad Ilyas, School of Mathematical and Physical Sciences, University of Newcastle, Callaghan, NSW 2308, Australia mailto:muhammad.ilyas@uon. edu. au

2. Bishnu P. Lamichhane, School of Mathematical and Physical Sciences, University of Newcastle, Callaghan, NSW 2308, Australia 